\title{
What Happens to Patients Discharged Against Medical Advice?
}

\author{
Nagarajan Ramakrishnan, Lakshmi Ranganathan, Babu K. Abraham, Senthilkumar Rajagopalan, Ramesh Venkataraman \\ Department of Critical Care Medicine, Apollo Hospitals, Chennai, Tamil Nadu, India
}

\section{Abstract}

Introduction: Discharge against medical advice (DAMA) when requested by family members of patients in Indian Critical Care Units (CCUs) leads to loss of follow-up and unknown patient outcomes. Exclusion of these patients from research analysis and quality audits confounds these results. We hence explored the proportion of patients leaving DAMA, reasons, and their outcomes at 30 and 90 days. Methods: This was a prospective study of CCU patients admitted from July 2013 to February 2014. All patients, who were DAMA during this period, were included in this study. Demographics, APACHE data, and outcomes were collected and compared to patients discharged regularly during the same period. Outcomes of DAMA patients at 30 and 90 days were gathered by telephone follow-up. Results: Among the 663 patients admitted to the CCU, 15.1\% (100 patients) were DAMA. The baseline APACHE score of the DAMA group was higher than the regular discharge group $(29.5 \pm 8.6$ vs. $26.1 \pm 10.3 ; P=0.002)$. During the 30 -day follow-up, $23 \%$ were alive, $56 \%$ dead, and $21 \%$ could not be contacted. At 90 days, mortality was $62 \%$ and survivors decreased to $14 \%$. Common reasons for DAMA were - wanting to take the patient to another hospital (21\%), reluctance for further treatments (12\%), financial constraints (9\%), and overall poor prognosis (7\%). Conclusion: A significant proportion of patients in the CCU get DAMA despite high severity of illness. Understanding the outcomes of these patients will help refine CCU quality audit reports and research study results.

Keywords: APACHE score, Critical Care Unit, discharge against medical advice, mortality, standard mortality rate

\section{INTRODUCTION}

Discharge against medical advice (DAMA) is not an uncommon request from family members of patients in Indian Critical Care Units (CCU). Patients who leave against medical advice are both a challenge and concern for physicians, ${ }^{[1]}$ as these patients are lost to follow-up, and their outcomes remain unknown. Demographic, social and clinical factors may be responsible for this phenomenon which is referred in several abbreviated forms such as DAMA (discharge against medical advice), SAMA (signing against medical advice), LAMA (leaving against medical advice), or DAOR (discharge at own risk). The phenomenon poses serious clinical, ethical, and legal challenges to the individual physician as well as the hospitals. ${ }^{[2]}$

Incidence, reasons, and outcome of DAMA have not been adequately studied but can have a significant impact on the evaluation of quality of care. ${ }^{[3]}$ The exclusion of these patients from analysis of research studies and quality audits such as calculation of standardized mortality ratio confounds results and reports. DAMA has also been shown to be associated with significantly higher risk of morbidity and mortality, hospital readmission, and higher costs for

\begin{tabular}{|l|l|}
\hline \multicolumn{2}{|c|}{ Access this article online } \\
\hline Quick Response Code: & Website: \\
\hline & www.ijccm.org \\
\hline & \\
\hline
\end{tabular}

the subsequent care of an initially inadequately treated condition. ${ }^{[4-6]}$

In this study, we seek to explore the proportion of patients DAMA from our multidisciplinary CCU, the reasons for DAMA decision, and their outcomes at days 30 and 90 .

\section{Methods}

\section{Study design}

This was a prospective case-control study conducted in a CCU in a tertiary care hospital in Chennai, from July 2013 to February 2014. All consecutive patients who left against medical advice from the medical-surgical CCU during the study were included in the study. AMA was defined as any patient who despite not being medically ready, insisted upon leaving against the advice of the treating medical team.

Address for correspondence: Dr. Nagarajan Ramakrishnan, Department of Critical Care Medicine, Apollo Hospitals, Chennai, Tamil Nadu, India. E-mail: ram@icuconsultants.com

This is an open access journal, and articles are distributed under the terms of the Creative Commons Attribution-NonCommercial-ShareAlike 4.0 License, which allows others to remix, tweak, and build upon the work non-commercially, as long as appropriate credit is given and the new creations are licensed under the identical terms.

For reprints contact: reprints@medknow.com

How to cite this article: Ramakrishnan N, Ranganathan L, Abraham BK, Rajagopalan S, Venkataraman R. What happens to patients discharged against medical advice? Indian J Crit Care Med 2018;22:580-4. 


\section{Data collection}

The study was part of ongoing quality improvement efforts. Patient demographics, APACHE II scores, admission diagnosis, and CCU length of stay (LOS) were collected. Details of payment mode was collected and categorized as self (paid by patient or family), institution (patient's health care costs partly or completely supported by an institution to which patient is affiliated with) or third party (medical insurance payments). Other social and clinical details which may be relevant to their decision for DAMA such as domiciliary information and whether they were previously admitted at another facility within the past month for the current or related ailment were also collected. The reasons for discharge AMA, when provided, were noted down. Admission demographic data were compared between patients who were discharged AMA and patients who were discharged on medical recommendation during the same period.

After their DAMA, the patients were followed up twice, initially at 30 days and subsequently at 90 days. These outcome data were collected through telephone calls to the family by a dedicated research coordinator.

\section{Data analysis}

Categorical variables are presented as frequencies and percentages. Characteristics of patients in the DAMA group were compared with those discharged regularly using Chi-square tests and Student's $t$-test as appropriate. $P<$ 0.05 was considered significant for all analyses. All other factors that were associated with DAMA were also analyzed. Statistical analysis employed SPSS software (SPSS ver. 17, IBM, Chicago, USA).

\section{RESULTS}

A total of 663 patients were admitted during the study period, among whom $100(15.08 \%)$ were DAMA. Of these $70 \%$ were males, $30 \%$ were females, $93 \%$ were medical admissions, and $7 \%$ were surgical admissions. Their mean age was $55.3 \pm 16.9$, mean APACHE score was $29.5 \pm 8.6$, and mean Intensive Care Unit length of stay (ICU LOS) was $9.6 \pm 13.4$ [Table 1].

DAMA was more common in males $(70 \%)$, and in the age group of $61-70$, constituting $25 \%$ of the total [Figure 1a]. General medicine and neurology admissions were more common among the DAMA group. Figure $1 \mathrm{~b}$ shows the common diagnoses of patients in the DAMA group.

Majority of the DAMA patients (49\%) were from Chennai where our hospital is located. Nearly $27 \%$ were from other parts of our state (Tamil Nadu), and 24\% were from out of state [Table 2].

\section{Reasons for discharge against medical advice}

Reasons for DAMA were documented at the time of discharge of the patient [Figure 2]. Response to this question was optional as some of the family members were unclear and/or were unwilling to provide the specific reason(s). Among the

\begin{tabular}{|c|c|c|c|}
\hline & DAMA & $\begin{array}{c}\text { Discharge } \\
\text { on medical } \\
\text { recommendation }\end{array}$ & Significance \\
\hline Total admission & 100 & 563 & \\
\hline \multicolumn{4}{|l|}{ Gender $(\%)$} \\
\hline Males & $70(70)$ & $354(63)$ & 0.91 \\
\hline Females & $30(30)$ & $209(37)$ & \\
\hline Mean age & $55.3 \pm 16.9$ & $56.4 \pm 16.4$ & 0.509 \\
\hline \multicolumn{4}{|l|}{ Source of admission } \\
\hline Emergency & $83(83)$ & $440(78)$ & 0.836 \\
\hline Wards & $9(9)$ & $32(6)$ & \\
\hline From another $\mathrm{CCU}$ & $8(8)$ & $91(16)$ & \\
\hline \multicolumn{4}{|l|}{ Type of admission } \\
\hline Medical & $93(93)$ & $477(85)$ & 0.724 \\
\hline Surgical & $7(7)$ & $86(15)$ & \\
\hline APACHE II score & $29.5 \pm 8.6$ & $26.12 \pm 10.3$ & 0.002 \\
\hline \multicolumn{4}{|l|}{ Ventilator status } \\
\hline Intubated & $82(82)$ & $339(60)$ & 0.518 \\
\hline Not intubated & $18(18)$ & $224(40)$ & \\
\hline CCU LOS & $9.6 \pm 13.4$ & $8.3 \pm 7.6$ & 0.214 \\
\hline
\end{tabular}

Table 2: Characteristics of patients in the discharge against medical advice group

\begin{tabular}{lc}
\hline Characteristic & Percentage of patients \\
\hline Total admission & 663 \\
Number of AMA (\%) & $100(15.08)$ \\
Hometown & \\
Chennai & 49 \\
Tamil Nadu & 24 \\
Other state & 27 \\
Previous hospitalization (within one month) & 58 \\
Readmission after DAMA to our hospital & 6 \\
\hline
\end{tabular}

DAMA: Discharge against medical advice; AMA: Against medical advice

family members of patients who were discharged AMA, 37\% did not want to disclose the reason, $21 \%$ wanted to take their family member to another hospital, and $12 \%$ were reluctant for further treatments such as surgery or what they perceived as complicated procedures. Financial constraint, however, was the primary reason for DAMA in $9 \%$. A small proportion (8\%) preferred to continue treatment at hospitals closer to home while others $(7 \%)$ decided on DAMA due to overall poor prognosis.

\section{Previous admissions and readmissions of discharge} against medical advice patients

Chart review of the DAMA patient group revealed that $57 \%$ of these patients had had a previous admission in either our hospital or at different facility within 30 days before this admission. After DAMA, six patients were readmitted to our hospital within a 3-month period. 


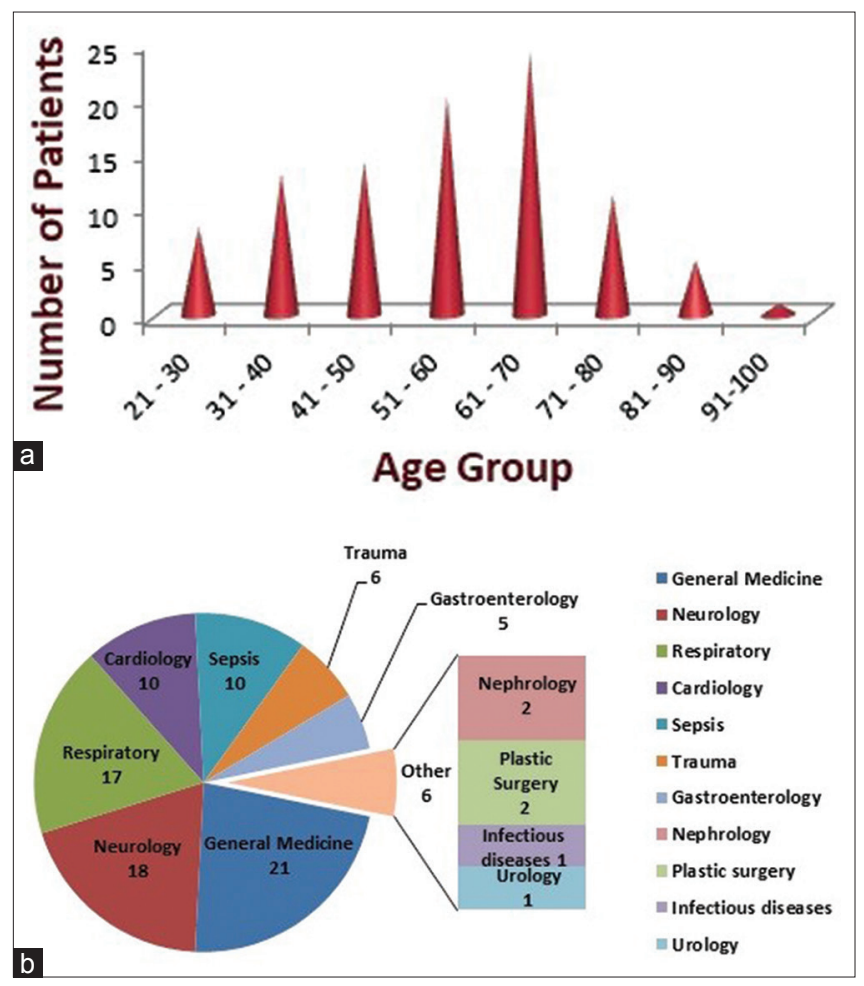

Figure 1: (a) Age distribution in the discharge against medical advice group. (b) Diagnosis in the discharge against medical advice group

\section{Comparisons between discharge against medical advice group and regular discharge (on medical advice) group}

Table 1 shows the comparison between CCU characteristics of patients who went DAMA and those discharged regularly. Patients in the DAMA group had a significantly higher APACHE score as compared to the normal discharge group $(P=0.002)$. It was noted that those discharged against medical advice were younger and had a higher proportion of males. The mean ICU length of stay (LOS) for the DAMA group was longer than for those with standard discharge (9.6 vs. 8.3 days). However, these differences were not statistically different between the groups.

\section{Outcome at 30 and 90 days}

During the follow-up at 30 days, 56 patients had expired, 23 were alive, and 21 were lost to follow-up. At 90 days, mortality was $62 \%$ and survivors decreased to $14 \%$.

\section{Discussion}

The phenomenon of DAMA is worldwide and is not limited to the developing countries. ${ }^{[1]}$ Despite the widespread nature of this problem, there has been little data evaluating incidence, reasons, and outcome of patients leaving DAMA. Studies have focused on specific patient groups such as patients admitted to the emergency department ${ }^{[7]}$ or psychiatric/orthopedic patients. ${ }^{[8]}$ We did not find any studies specifically evaluating this in critically ill patients in India.

Incidence of DAMA seems to vary depending on the patient population and treatment setting. ${ }^{[7]}$ Studies have shown that

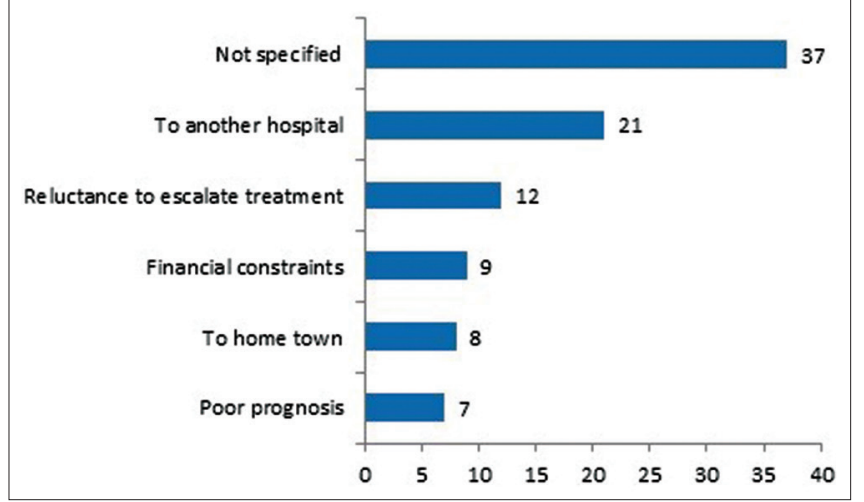

Figure 2: Reasons for discharge against medical advice

there is considerable variation in the prevalence rate of DAMA, ranging from $0.7 \%$ to $2.2 \%$, but may be as high as $25.9 \%$ in some centers. ${ }^{[2,4]} \mathrm{Al}$ Ayed, in his review paper, has reported varied incidence of DAMA ranging from $>20 \%$ in large urban hospitals to $<4 \%$ in small rural hospitals and medical wards. ${ }^{[1]}$ Some studies have documented a higher rate of DAMA in developing than developed countries. ${ }^{[8]}$ Although our center, DAMA rates are slightly lower to that of rates for a large urban hospital quoted by Al Ayed, ${ }^{[1]}$ our patients may be socioeconomically and culturally different than the patients in his study. We do not have any data for similar comparison from within India.

Prior studies have shown that DAMA patients were younger ${ }^{[6]}$ and predominantly males. ${ }^{[6,9-11]}$ However, other studies have provided similar results to ours and have found no association between patient age and DAMA. ${ }^{[12]}$ Some authors hypothesize that the reason for more males in the DAMA group could be due to their social responsibility in caring for their family and due to their risk-taking attitude in making decisions; ${ }^{[10]}$ however, this may not be applicable to the critical care setting, where the patient is often medically incapacitated and unable to make decisions. According to the review by David, common factors that have been consistently associated with DAMA include young age, male gender, poor social support, and lack of health care coverage. ${ }^{[4,5,8,9,13]}$ Psychiatric illness and substance abuse are other reasons often cited for DAMA. ${ }^{[8,14-16]}$ Geographic reasons and proximity of care to home may also be factors leading to DAMA. ${ }^{[17]}$

Contrary to the finding of studies which showed that ICU LOS was shorter for patients leaving $\mathrm{AMA},{ }^{[6,8]}$ we could not demonstrate any such difference. It is possible that our study was underpowered to show such a difference or our DAMA practices differed from that of other centers.

Among all the reasons mentioned for DAMA, financial constraints and lack of financial support were reported as common and personal factor contributing to DAMA in several studies. ${ }^{[2,18,19]}$ Jimoh et al., in their study on AMA from a medical center in Abuja, recorded 33\% DAMAs due to financial reasons. ${ }^{[2]}$ The authors opine that, it could be due to widespread poverty and lack of access to health insurance by 
the majority of people, that could have been the key contributor for DAMA. An Indian study has explored the reasons for patients leaving AMA from the emergency department, where $84 \%$ reported leaving because of financial restrictions. ${ }^{[7]}$ In our study, only $9 \%$ patients quoted financial constraints as the reason for DAMA. This could likely be a falsely low rate considering $37 \%$ of patients who left DAMA in our study did not want to specify the reason for DAMA, and a proportion of them could have left unable to bear the costs of treatment. In addition, $21 \%$ of respondents mentioned that they wanted to take their ward to another hospital and could have moved to a hospital that was less expensive. As has been shown in several studies that DAMA patients are more often economically disadvantaged and lack health insurance, ${ }^{[6,11,13,17,20]}$ none of the patients in our study had health insurance, but a small proportion (19\%) patients had some coverage through their employer.

Other reasons commonly cited for AMA were perceived improvement in clinical state, personal issues, preference for other hospitals or continuing treatment at home, and dissatisfaction with hospital services. ${ }^{[13,18,21-23]}$ Some studies have also shown unspecified reasons, ${ }^{[2]}$ and some have shown more than one reason. ${ }^{[10]}$ In our study, majority (37\%) did not specify reasons; however, none had mentioned more than one reason. Furthermore, DAMA patients have shown to prefer alternative therapy for multiple reasons including fear of surgery and possibility of resolution of their problems both physically and spiritually. ${ }^{[2]}$ In our study, $12 \%$ were reluctant for a surgery or a complicated procedure and $7 \%$ left due to the poor prognosis conveyed by the clinical team.

It is often assumed that patients who leave against medical advice reject medical care. Paradoxically, it is shown that these patients are frequently readmitted to the same hospital. ${ }^{[1]}$ Those who left AMA were more likely to be readmitted, within 15-30 days, often for the same problems that precipitated the initial admissions..$^{[5,6,23]}$ In our study, only $6 \%$ patients were readmitted to our hospital. This could be explained by the fact that DAMA was done in the first place due to anticipated poor outcome, transfer of care to a hospital near home, or due to financial constraints.

We also collected the information on previous admission detail of the DAMA patients and found that $57 \%$ of patients were previously admitted to our or another hospital within 30 days of this admission. It is plausible that the previous admission could have impacted the patients' family financially and emotionally leading to DAMA during this admission.

Although it would be rational to expect that only individuals with less life threatening conditions would leave DAMA, there are several reports of patients with very serious diagnoses who have left against medical advice..$^{[1]}$ In our study, patients who left DAMA had a very high severity of illness, and perception of a poor outcome by the caregivers and the family could have influenced their decision to DAMA. While a moderately sized prospective study found no relationship between AMA discharge and death, ${ }^{[23]}$ several other studies, found a high rate of mortality among patients who were discharged AMA. ${ }^{[24]}$ One study found that DAMA patients had $60 \%$ higher risk for death. ${ }^{[6]}$ In our study, in the one month follow-up, 56 (56\%) patients had expired. By the end of 3 months, this number increased to $62(62 \%)$.

To the best of our knowledge, this is the first prospective study with long-term follow-up that has studied DAMA in Critical Care Setting in India. This study has thrown light on important factors affecting and reasons leading to DAMA.

Our study has several potential limitations. Our center is an urban tertiary care hospital and DAMA rates here may not reflect the practices across the region or country. In addition, almost $75 \%$ of the DAMA patients were from our state (Tamil Nadu), and hence, the DAMA rates could have been higher. We did not mandate the response for reason for DAMA from families and are limited in that $37 \%$ of DAMA in our study happened without any reasons recorded. This could have confounded our results.

\section{ConcLusion}

A significant proportion of patients in the CCU leave against medical advice for varied reasons despite high severity of illness. Contrary to common perception, $23 \%$ of these patients are alive at 3 months' follow-up. Transfer to another facility to continue care was the most common reason for DAMA. Understanding the outcomes of these patients better will help refine CCU quality audit reports and research study results in the future.

\section{Financial support and sponsorship}

Nil.

\section{Conflicts of interest}

There are no conflicts of interest.

\section{References}

1. Al Ayed I. What makes patients leave against medical advice? J Taibah Univ Med Sci 2009;4:16-22.

2. Jimoh BM, Anthonia OC, Chinwe I, Oluwafemi A, Ganiyu A, Haroun A, et al. Prospective evaluation of cases of discharge against medical advice in Abuja, Nigeria. ScientificWorldJournal 2015;2015:314817.

3. Ba L, Zhang M, Su L, Cheng Z, Xu Y. Nine-year change of mortality and discharge against medical advice among major trauma patients in a Chinese Intensive Care Unit. Eur J Trauma Emerg Surg 2016;42:47-53.

4. Saitz R, Ghali WA, Moskowitz MA. The impact of leaving against medical advice on hospital resource utilization. J Gen Intern Med 2000;15:103-7.

5. Aliyu ZY. Discharge against medical advice: Sociodemographic, clinical and financial perspectives. Int J Clin Pract 2002;56:325-7.

6. Fiscella K, Meldrum S, Barnett S. Hospital discharge against advice after myocardial infarction: Deaths and readmissions. Am J Med 2007;120:1047-53.

7. Naderi S, Acerra JR, Bailey K, Mukherji P, Taraphdar T, Mukherjee T, et al. Patients in a private hospital in India leave the emergency department against medical advice for financial reasons. Int $\mathrm{J}$ Emerg Med 2014;7:13.

8. Seaborn Moyse H, Osmun WE. Discharges against medical advice: A community hospital's experience. Can J Rural Med 2004;9:148-53. 
9. Baptist AP, Warrier I, Arora R, Ager J, Massanari RM. Hospitalized patients with asthma who leave against medical advice: Characteristics, reasons, and outcomes. J Allergy Clin Immunol 2007;119:924-9.

10. Noohi K, Komsari S, Nakhaee N, Yazdi Feyzabadi V. Reasons for discharge against medical advice: A case study of emergency departments in Iran. Int J Health Policy Manag 2013;1:137-42.

11. Weingart SN, Davis RB, Phillips RS. Patients discharged against medical advice from a general medicine service. J Gen Intern Med 1998;13:568-71.

12. Shirani F, Jalili M, Asl-E-Soleimani H. Discharge against medical advice from emergency department: Results from a tertiary care hospital in Tehran, Iran. Eur J Emerg Med 2010;17:318-21.

13. Alfandre DJ. "I'm going home": Discharges against medical advice. Mayo Clin Proc 2009;84:255-60.

14. Dalrymple AJ, Fata M. Cross-validating factors associated with discharges against medical advice. Can J Psychiatry 1993;38:285-9.

15. Green P, Watts D, Poole S, Dhopesh V. Why patients sign out against medical advice (AMA): Factors motivating patients to sign out AMA. Am J Drug Alcohol Abuse 2004;30:489-93.

16. Franks P, Meldrum S, Fiscella K. Discharges against medical advice: Are race/ethnicity predictors? J Gen Intern Med 2006;21:955-60.

17. Karimi SA, Saravi BM, Farahabbadi EB, Zamanfar D, Fallah M,
Abokheily MA, et al. Studying the rate and causes of discharge against medical advice in hospitals affiliated to mazandaran university of medical sciences. Mater Sociomed 2014;26:203-7.

18. Fatemeh RJ, Maryam RJ, Rezaeiimofrad M. Patients' reasons for discharge against medical advice in university hospitals of Kashan university of medical sciences in 2008. Hakim Health Sys Res 2010;13:33-9.

19. Ikefuna A, Emodi I. An assessment of factors influencing hospital discharges against medical advice of paediatric patients in enugu: A review of 67 cases. Niger J Paediatr 2002;29:1-4.

20. Ding R, Jung JJ, Kirsch TD, Levy F, McCarthy ML. Uncompleted emergency department care: Patients who leave against medical advice. Acad Emerg Med 2007;14:870-6.

21. Nasir AA, Babalola OM. Clinical spectrum of discharges against medical advice in a developing country. Indian J Surg 2008;70:68-72.

22. Tabatabaei SM, Sargazi Moakhar Z, Behmanesh Pour F, Shaare Mollashahi S, Zaboli M. Hospitalized pregnant women who leave against medical advice: Attributes and reasons. Matern Child Health J 2016;20:128-38

23. Hwang SW, Li J, Gupta R, Chien V, Martin RE. What happens to patients who leave hospital against medical advice? CMAJ 2003;168:417-20.

24. Link K, Brody CE, Chan J. Leaving a medical service against advice. Va Med 1983;110:100-2. 